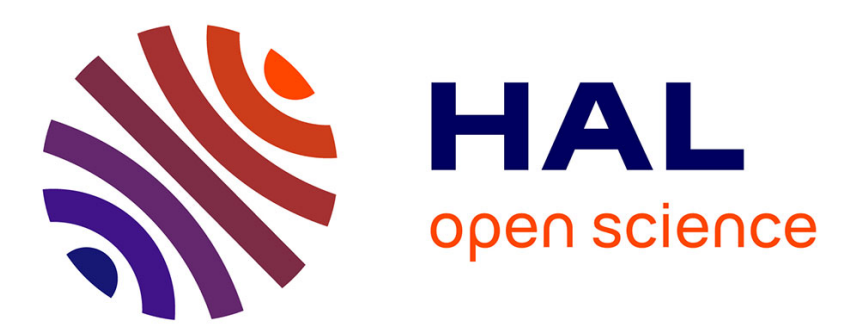

\title{
Inertial-aided Homography-based Visual Servo Control of Autonomous Underwater Vehicles without Linear Velocity Measurements
}

\author{
Lam-Hung Nguyen, Minh Duc Hua, Guillaume Allibert, Tarek Hamel
}

\section{- To cite this version:}

Lam-Hung Nguyen, Minh Duc Hua, Guillaume Allibert, Tarek Hamel. Inertial-aided Homographybased Visual Servo Control of Autonomous Underwater Vehicles without Linear Velocity Measurements. ICSTCC, Oct 2017, Sinaia, Romania. hal-01627666

\section{HAL Id: hal-01627666 \\ https://hal.science/hal-01627666}

Submitted on 2 Nov 2017

HAL is a multi-disciplinary open access archive for the deposit and dissemination of scientific research documents, whether they are published or not. The documents may come from teaching and research institutions in France or abroad, or from public or private research centers.
L'archive ouverte pluridisciplinaire HAL, est destinée au dépôt et à la diffusion de documents scientifiques de niveau recherche, publiés ou non, émanant des établissements d'enseignement et de recherche français ou étrangers, des laboratoires publics ou privés. 


\title{
Inertial-aided Homography-based Visual Servo Control of Autonomous Underwater Vehicles without Linear Velocity Measurements
}

\author{
Lam-Hung Nguyen, Minh-Duc Hua, Guillaume Allibert, Tarek Hamel \\ Université Côte d'Azur, CNRS, I3S \\ Sophia Antipolis, France \\ lhnguyen(hua,allibert,thamel)@i3s.unice.fr
}

\begin{abstract}
An inertial-aided visual servo control approach for fully-actuated Autonomous Underwater Vehicles (AUVs) without relying on linear velocity measurements is proposed. The homography obtained from corresponding images of a locally planar scene is directly exploited as feedback information. A cascade inner-outer loop control architecture is adopted that facilitates both control implementation and gain tuning procedures. Control robustness with respect to model uncertainties and external disturbances is reinforced using both high gain estimation and integrator techniques. Rigourous stability analysis and insightful discussions are provided throughout the paper. Finally, robustness and efficiency of the proposed approach are illustrated by simulation on a realistic AUV model.
\end{abstract}

Keywords-AUV, visual servoing, homography-based control, nonlinear control

\section{INTRODUCTION}

Safe and efficient navigation of robotic underwater vehicles in cluttered areas (near man-made infrastructures or the close to the ocean floor) remains a big challenge. Scientific issues are mainly related to the fact that global acoustic positioning systems become unusable or insufficiently precise in such situations. Therefore, the AUV must rely on exteroceptive sensors and advanced sensor-based control strategies. Although sensing the surrounding environment can be done by various types of sensors, cameras remain an attractive solution. By using camera(s) as a sensor modality for relative position and orientation, the control problem can be cast into PositionBased Visual Servo (PBVS) or Image-Based Visual Servo (IBVS) control problems [4]. Classical visual servo control approaches have been proposed for robotic manipulators and mobile ground vehicles (see [4]) and then for aerial drones [7], [19]. In underwater robotics, vision sensors have been used to perform tasks such as pipeline following [12], [20], station keeping [15], or positioning [6]. Both stereo and monocular visions have been exploited for the problems of stabilization and positioning of AUVs. When it is possible to reconstruct the vehicle's pose (i.e. position and orientation), existing positionbased controllers can be directly applied [18]. The case of monocular vision without the assumption of planarity of the visual target and the prior knowledge of its geometry is more challenging since the pose cannot be fully reconstructed from visual data. However, similarly to the case of robotic manipulators and aerial robots [4], [19], monocular vision can be sufficient to achieve stabilization for AUVs in front of a planar target using $2 \frac{1}{2} \mathrm{D}$ servoing [3], [15], [21], essentially based on the idea of Malis et al. [16]. Recently, in [2] an advanced kinematic IBVS control scheme was developed by exploiting the so-called homography matrix. A noticeable feature of that approach is that the step of homography decomposition [17] often computationally expensive is not required as opposed to other homography-based visual servo (HBVS) controllers [16]. More recently, this kinematic HBVS control approach has been extended in our prior work [9], [13] in order to account for the full dynamics of fully-actuated AUVs and to obtain an enlarged provable domain of convergence. The present paper is the continuation of our prior work on the same topic of dynamic HBVS control of fully-actuated AUVs, but here without relying on linear velocity measurements. A motivation behind this effort is linked to the high price and high weight of Doppler Velocity Log (DVL) velocity sensors commonly used for AUV navigation. Moreover, many applications such as intervention and high-resolution imaging for inspection require the vehicle to operate in close proximity to man-made infrastructures (about $1 \mathrm{~m}$ ). However, at such a close distance the maximum slope-threshold of a DVL can be easily violated and DVL measurements then become very imprecise, thereby resulting in an obvious need of advanced vision-based control techniques independent of linear velocity measurements.

The paper is organized as follows. Notation and system modelling are given in Section II. Problem formulation of HBVS control of fully-actuated AUVs is presented in Section III. Section IV presents a novel inertial-aided HBVS control approach for fully-actuated and compact AUVs. Section V reports simulation results on a realistic AUV model showing the performance and robustness of the proposed algorithm. A short conclusion then follows.

\section{SYSTEM MODELLING}

\section{A. Notation}

- $\left\{\mathbf{e}_{1}, \mathbf{e}_{2}, \mathbf{e}_{3}\right\}$ denotes the canonical basis of $\mathbb{R}^{3}$. $\mathbf{I}_{3}$ denotes the identity matrix of $\mathbb{R}^{3 \times 3}$. The notation $\mathbf{u}_{\times}, \forall \mathbf{u} \in \mathbb{R}^{3}$, denotes the skew-symmetric matrix associated with the cross product by $\mathbf{u}$, i.e., $\mathbf{u}_{\times} \mathbf{v}=\mathbf{u} \times \mathbf{v}, \forall \mathbf{v} \in \mathbb{R}^{3}$. The operator $\operatorname{vex}(\cdot)$ is defined such that $\operatorname{vex}\left(\mathbf{u}_{\times}\right)=\mathbf{u}, \forall \mathbf{u} \in \mathbb{R}^{3}$. 


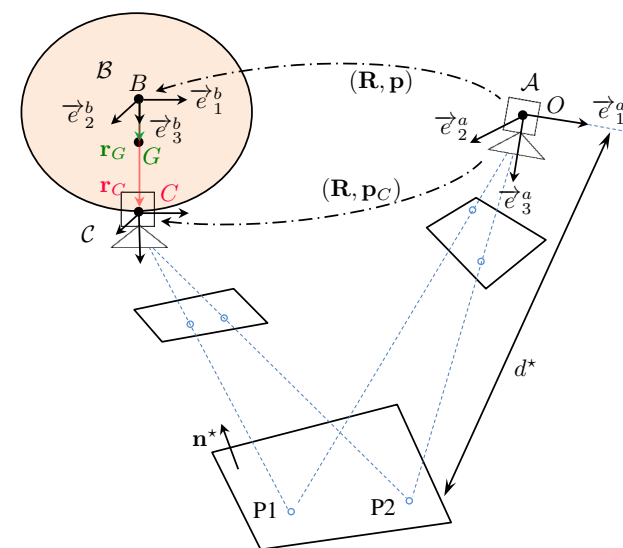

Fig. 1. A compact AUV with a downward-looking camera for homography estimation and notation

The Euclidean norm in $\mathbb{R}^{n}$ and the transpose operator are, respectively, denoted as $|\cdot|$ and $(\cdot)^{\top}$. Let $\operatorname{sat}^{\delta}(\cdot) \in \mathbb{R}^{n}$, with $\delta>0$, be the classical saturation function defined by $\operatorname{sat}^{\delta}(\mathbf{x}) \triangleq \mathbf{x} \min (1, \delta /|\mathbf{x}|), \forall \mathbf{x} \in \mathbb{R}^{n}$.

- Let $G$ and $B$ denote the AUV's center of mass (CoM) and center of buoyancy $(\mathrm{CoB}), m$ its mass and $\mathbf{J}_{0}$ its inertia matrix. The distance between $G$ and $B$ is denoted as $l$.

- Let $\mathcal{A}=\left\{O ; \vec{e}_{1}^{a}, \vec{e}_{2}^{a}, \vec{e}_{3}^{a}\right\}$ and $\mathcal{B}=\left\{B ; \vec{e}_{1}^{b}, \vec{e}_{2}^{b}, \vec{e}_{3}^{b}\right\}$ denote an inertial frame and a frame attached to the AUV. Let $\mathcal{C}=\left\{C ; \vec{e}_{1}^{c}, \vec{e}_{2}^{c}, \vec{e}_{3}^{c}\right\}$ be a frame attached to the camera, with its base vectors parallel to those of $\mathcal{B}$. In this paper we assume that $B, G, C$ are aligned (Fig. 1). Let $\mathbf{r}_{C}=l_{C} \mathbf{e}_{3} \in$ $\mathbb{R}^{3}$ and $\mathbf{r}_{G}=l \mathbf{e}_{3} \in \mathbb{R}^{3}$, with $\mathbf{e}_{3}=[0,0,1]^{\top}$, denote the vectors of coordinates expressed in the frame $\mathcal{B}$ of $\overrightarrow{B C}$ and $\overrightarrow{B G}$, respectively.

- The orientation of $\mathcal{B}$ relative to $\mathcal{A}$ is represented by the rotation matrix $\mathbf{R} \in \mathrm{SO}(3)$. Denote the position of the origins of the frames $\mathcal{B}$ and $\mathcal{C}$, expressed in $\mathcal{A}$, as $\mathbf{p}$ and $\mathbf{p}_{C}$, respectively. We have $\mathbf{p}=\mathbf{p}_{C}-\mathbf{R r}_{C}$

- The angular velocity, expressed in $\mathcal{B}$, of the frame $\mathcal{B}$ with respect to (w.r.t.) $\mathcal{A}$ is denoted as $\boldsymbol{\Omega}=\left[\omega_{1}, \omega_{2}, \omega_{3}\right]^{\top} \in \mathbb{R}^{3}$. The linear velocity of $B$ and $C$, expressed in the frame $\mathcal{B}$, are $\mathbf{V} \in \mathbb{R}^{3}$ and $\mathbf{V}_{C} \in \mathbb{R}^{3}$. We have $\mathbf{V}=\mathbf{V}_{C}-\mathbf{\Omega} \times \mathbf{r}_{C}$.

- Let $\mathbf{v}_{f}$ and $\mathbf{V}_{f}$ denote the current velocity expressed in the frames $\mathcal{A}$ and $\mathcal{B}$, respectively. In this paper we assume that $\mathbf{v}_{f}$ is constant. Denote $\mathbf{V}_{h} \triangleq \mathbf{V}-\mathbf{V}_{f}$ as the CoB's velocity w.r.t. the current.

\section{B. Recalls on system modeling}

Define $\mathbf{W}_{h} \triangleq\left[\mathbf{V}_{h}^{\top}, \boldsymbol{\Omega}^{\top}\right]^{\top} \in \mathbb{R}^{6}$. The total kinetic energy of the body-fluid system $E_{T}$ is defined as the sum of the kinetic energy of the vehicle $E_{B}$ and the one of the surrounding fluid $E_{F}$, i.e. $E_{T_{1}}=E_{B}+E_{F}$ with

$$
\begin{gathered}
E_{B}=\frac{1}{2} \mathbf{W}_{h}^{\top} \mathbf{M}_{B} \mathbf{W}_{h}, \text { with } \mathbf{M}_{B} \triangleq\left[\begin{array}{cc}
m \mathbf{I}_{3} & -m \mathbf{r}_{G \times} \\
m \mathbf{r}_{G \times} & \mathbf{J}_{0}
\end{array}\right] \\
E_{F}=\frac{1}{2} \mathbf{W}_{h}^{\top} \mathbf{M}_{A} \mathbf{W}_{h}, \text { with } \mathbf{M}_{A} \triangleq\left[\begin{array}{ll}
\mathbf{M}_{A}^{11} & \mathbf{M}_{A}^{12} \\
\mathbf{M}_{A}^{21} & \mathbf{M}_{A}^{22}
\end{array}\right]
\end{gathered}
$$

$\mathbf{M}_{A} \in \mathbb{R}^{6 \times 6}$ is referred to as the added mass matrix, which is approximately constant and symmetric [5]. Thus,

$$
E_{T}=\frac{1}{2} \mathbf{W}_{h}^{\top} \mathbf{M}_{T} \mathbf{W}_{h}, \text { with } \mathbf{M}_{T}=\left[\begin{array}{cc}
\mathbf{M} & \mathbf{D}^{\top} \\
\mathbf{D} & \mathbf{J}
\end{array}\right]
$$

with $\mathbf{M} \triangleq m \mathbf{I}_{3}+\mathbf{M}_{A}^{11}, \mathbf{J} \triangleq \mathbf{J}_{0}+\mathbf{M}_{A}^{22}, \mathbf{D} \triangleq m \mathbf{r}_{G \times}+\mathbf{M}_{A}^{21}$. The translational and rotational momentums are derived as

$$
\left\{\begin{array}{l}
\mathbf{P}_{h}=\frac{\partial E_{T}}{\partial \mathbf{V}_{h}}=\mathbf{M} \mathbf{V}_{h}+\mathbf{D}^{\top} \boldsymbol{\Omega} \\
\mathbf{\Pi}_{h}=\frac{\partial E_{T}}{\partial \boldsymbol{\Omega}}=\mathbf{J} \boldsymbol{\Omega}+\mathbf{D} \mathbf{V}_{h}
\end{array}\right.
$$

The vehicle's equations of motion are [14]

$$
\begin{aligned}
\dot{\mathbf{p}} & =\mathbf{R V} \\
\dot{\mathbf{R}} & =\mathbf{R} \boldsymbol{\Omega}_{\times} \\
\dot{\mathbf{P}}_{h} & =\mathbf{P}_{h} \times \boldsymbol{\Omega}+\mathbf{F}_{c}+\mathbf{F}_{g b}+\mathbf{F}_{d} \\
\dot{\boldsymbol{\Pi}}_{h} & =\boldsymbol{\Pi}_{h} \times \mathbf{\Omega}+\mathbf{P}_{h} \times \mathbf{V}_{h}+\boldsymbol{\Gamma}_{c}+\boldsymbol{\Gamma}_{g}+\boldsymbol{\Gamma}_{d}
\end{aligned}
$$

where $\mathbf{F}_{c} \in \mathbb{R}^{3}$ and $\boldsymbol{\Gamma}_{c} \in \mathbb{R}^{3}$ are the force and torque control inputs, $\mathbf{F}_{g b} \triangleq\left(m g-F_{b}\right) \mathbf{R}^{\top} \mathbf{e}_{3}$ is the sum of the gravity and buoyancy forces, $\boldsymbol{\Gamma}_{g} \triangleq m g l \mathbf{e}_{3} \times \mathbf{R}^{\top} \mathbf{e}_{3}$ is the gravity torque, and the damping force and torque $\mathbf{F}_{d}$ and $\boldsymbol{\Gamma}_{d}$ are modeled as

$$
\left\{\begin{array}{l}
\mathbf{F}_{d}\left(\mathbf{V}_{h}\right)=-\left(\mathbf{D}_{V l}+\left|\mathbf{V}_{h}\right| \mathbf{D}_{V q}\right) \mathbf{V}_{h} \\
\boldsymbol{\Gamma}_{d}(\boldsymbol{\Omega})=-\left(\mathbf{D}_{\Omega l}+|\boldsymbol{\Omega}| \mathbf{D}_{\Omega q}\right) \boldsymbol{\Omega}
\end{array}\right.
$$

with positive damping matrices $\mathbf{D}_{V l}, \mathbf{D}_{V q}, \mathbf{D}_{\Omega l}, \mathbf{D}_{\Omega q}$.

\section{Model for control design}

For control design purposes, some simplifications are considered.

- Equations (3c)-(3d) indicate that the translational and rotational dynamics are coupled, via the coupling matrix D. Such coupling dynamics complicate the control design process because for the control problem at hand linear velocity measurements $\left(\mathbf{V}\right.$ or $\left.\mathbf{V}_{h}\right)$ are not available. However, we limit our study to a class of compactshape AUVs so that the matrix $\mathbf{M}_{A}^{21}$ is negligible w.r.t. the diagonal elements of $\mathbf{M}_{A}$. Moreover, it is assumed that the distance between the vehicle's CoB and CoM and the vehicle's mass are relatively small so that the term $m \mathbf{r}_{G \times}$ involved in the definition of $\mathbf{D}$ is also small. Consequently, the derivative terms $\mathbf{D}^{\top} \dot{\boldsymbol{\Omega}}$ and $\mathbf{D} \dot{\mathbf{V}}_{h}$ involved, respectively, in the left-hand side of (3c) and (3d) can be neglected and considered as disturbance terms.

- Since $\mathbf{V}_{h}$ is not measured, the term $\left(\mathbf{M V} \mathbf{V}_{h}\right) \times \mathbf{V}_{h}$ involved in (3d) and often referred to as "Munk moment" in the literature is neglected in the control model. This simplification is acceptable for vehicles in compact form (such as box-shaped or spherical AUVs) and for slow motion ( $\mathbf{V}_{h}$ remaining relatively small).

- All unmeasured terms involving the current velocity $\mathbf{V}_{f}$ are regrouped and considered as disturbance terms.

Under these justifications, control design will be carried out on the basis of the following simplified control model

$$
\begin{aligned}
\dot{\mathbf{p}} & =\mathbf{R V} \\
\dot{\mathbf{R}} & =\mathbf{R} \boldsymbol{\Omega}_{\times} \\
\mathbf{M} \dot{\mathbf{V}} & =(\mathbf{M V}) \times \boldsymbol{\Omega}+\mathbf{F}_{c}+\mathbf{F}_{g b}+\mathbf{F}_{d}(\mathbf{V})+\boldsymbol{\Delta}_{F} \\
\mathbf{J} \dot{\boldsymbol{\Omega}} & =(\mathbf{J} \boldsymbol{\Omega}) \times \boldsymbol{\Omega}+\boldsymbol{\Gamma}_{c}+\boldsymbol{\Gamma}_{g}+\boldsymbol{\Delta}_{\Gamma}
\end{aligned}
$$

with the "disturbance" terms

$$
\begin{aligned}
\boldsymbol{\Delta}_{F}= & -\left(\mathbf{M} \mathbf{V}_{f}\right)_{\times} \boldsymbol{\Omega}-\mathbf{M} \boldsymbol{\Omega}_{\times} \mathbf{V}_{f}+\left(\mathbf{D}^{\top} \boldsymbol{\Omega}\right)_{\times} \boldsymbol{\Omega}-\mathbf{D}^{\top} \dot{\boldsymbol{\Omega}} \\
& +\mathbf{F}_{d}\left(\mathbf{V}_{h}\right)-\mathbf{F}_{d}(\mathbf{V}) \\
\boldsymbol{\Delta}_{\Gamma}= & \left(\mathbf{D} \mathbf{V}_{h}\right) \times \boldsymbol{\Omega}+\mathbf{P}_{h} \times \mathbf{V}_{h}-\mathbf{D} \dot{\mathbf{V}}_{h}+\boldsymbol{\Gamma}_{d}
\end{aligned}
$$


Since the control objective is fixed-point stabilization, the terms $\boldsymbol{\Delta}_{F}$ and $\boldsymbol{\Delta}_{\Gamma}$ would eventually converge to constant values. Therefore, these terms will be considered as constant and compensated via integral actions.

\section{PROBLEM FORMULATION}

\section{A. Problem formulation}

Based on a reference image, taken at some desired pose using a downward-looking monocular camera, and the current images, the control design objective consists in stabilizing the camera's pose to the reference one. Let us choose the inertial frame $\mathcal{A}$ attached to the camera's desired pose (see Fig. 1). Assume that we dispose of the estimation of the homography matrix $\mathbf{H}$, which contains geometric information about the rotation and translation between the current camera frame $\mathcal{C}$ and the reference camera frame $\mathcal{A}$ [2]

$$
\mathbf{H}=\mathbf{R}^{\top}-\frac{1}{d^{\star}} \mathbf{R}^{\top} \mathbf{p}_{C} \mathbf{n}^{\star \top}
$$

with $d^{\star}$ the distance between the camera optical center and the target plane (i.e. depth), and $\mathbf{n}^{\star} \in S^{2}$ the unit vector normal to the target plane expressed in $\mathcal{A}$ (see Fig. 1). The dynamics of $\mathbf{H}$ satisfy

$$
\dot{\mathbf{H}}=-\boldsymbol{\Omega}_{\times} \mathbf{H}-\frac{1}{d^{\star}} \mathbf{V}_{C} \mathbf{n}^{\star \top}
$$

In addition to the estimation of $\mathbf{H}$, it is assumed that an Inertial Measurement Unit (IMU) is available to provide measurements of the angular velocity $\Omega$ together with an approximate of the gravity direction $\mathbf{R}^{\top} \mathbf{e}_{3}$.

The control objective can be stated as the stabilization of $\mathbf{H}$ about the identity matrix $\mathbf{I}_{3}$, or equivalently the stabilization of $\left(\mathbf{R}, \mathbf{p}_{C}\right)$ about $\left(\mathbf{I}_{3}, \mathbf{0}\right)$ using $\mathbf{H}, \boldsymbol{\Omega}$ and $\mathbf{R}^{\top} \mathbf{e}_{3}$ as measurements. The difficulties of dynamic HBVS control design are related to the fact that both $d^{\star}$ and $\mathbf{n}^{\star}$ involved in the expression (6) of $\mathbf{H}$ are unknown and that $\mathbf{H}$ only contains a coupled information of rotation and translation. More importantly, the linear velocity is not measured.

\section{B. Discussions on existing HBVS control approaches}

We first discuss about the state-of-the-art kinematic HBVS control proposed in [2]. The authors in [2] define the visual errors $\mathbf{e}_{p}, \mathbf{e}_{\Theta} \in \mathbb{R}^{3}$ as

$$
\mathbf{e}_{p} \triangleq\left(\mathbf{I}_{3}-\mathbf{H}\right) \mathbf{m}^{\star}, \quad \mathbf{e}_{\Theta} \triangleq \operatorname{vex}\left(\mathbf{H}^{\top}-\mathbf{H}\right)
$$

with some arbitrary unit vector $\mathbf{m}^{\star} \in S^{2}$ satisfying the following assumption $\mathbf{n}^{\star \top} \mathbf{m}^{\star}>0$. Note that in practice such an assumption is not restrictive. Indeed, since the visual target is observed by the camera at the reference pose, the angle between the target plane's normal vector and the opposite direction of the camera axis must be in the domain $[0, \pi / 2)$. Considering the fact that the camera points downwards as in the present paper, one has $\mathbf{n}^{\star \top}\left(-\mathbf{e}_{3}\right)>0$. This suggests the choice of $\mathbf{m}^{\star}=-\mathbf{e}_{3}$ without any knowledge about $\mathbf{n}^{\star}$.

Lemma 1 (See [2] for the proof) Under assumption $\mathbf{n}^{\star \top} \mathbf{m}^{\star}>0$, the kinematic control law:

$$
\mathbf{V}_{C}=-k_{p} \mathbf{e}_{p}, \quad \mathbf{\Omega}=-k_{\Theta} \mathbf{e}_{\Theta}
$$

with $k_{p}, k_{\Theta}>0$, ensures the local exponential stability of the equilibrium $\left(\mathbf{R}, \mathbf{p}_{C}\right)=\left(\mathbf{I}_{3}, \mathbf{0}\right)$, i.e. $\mathbf{H}=\mathbf{I}_{3}$. The function $\mathbf{e} \triangleq\left[\mathbf{e}_{p}^{\top}, \mathbf{e}_{\Theta}^{\top}\right]^{\top}$ is isomorphic to $\mathbf{H}$, i.e. $\mathbf{e}=\mathbf{0}$ iff $\mathbf{H}=\mathbf{I}_{3}$.
For mechanical systems, control inputs for control design should be forces and torques, instead of the angular and linear velocities. However, the passage from kinematic control to dynamic control requires both linear velocity and angular velocity measurements (see [9] for more extensive discussions) and is, thus, inapplicable to the problem considered in this paper due to the unavailability of linear velocity measurements.

In our prior work [9] concerning dynamic HBVS control, although significant efforts have been made to extend the domain of stability to (almost) global asymptotical stability, the proposed control approach still necessitates linear velocity measurements. Therefore, a novel dynamic HBVS control approach without relying on linear velocity measurements will be developed in the sequel.

\section{CONTROL DESIGN}

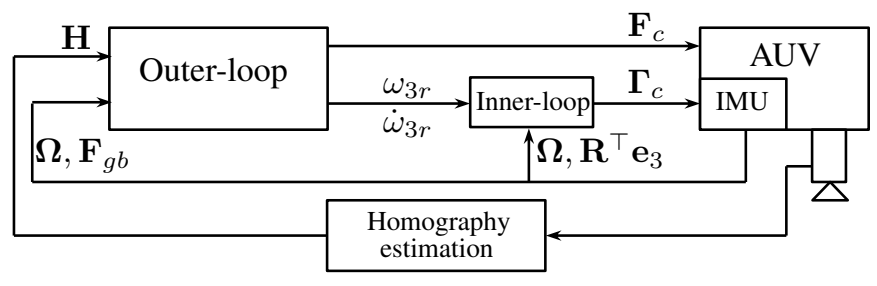

Fig. 2. Control architecture of the proposed HBVS

The following cascade inner-outer loop control architecture (illustrated by Fig. 2) is adopted:

- The inner-loop control, governing the rotation dynamics (5b) and (5d), defines the torque control vector $\boldsymbol{\Gamma}_{c}$ so as to ensure the asymptotic stabilization of $\left(\boldsymbol{\Omega}, \mathbf{R}^{\top} \mathbf{e}_{3}\right)$ about $\left(\boldsymbol{\Omega}_{r}, \mathbf{e}_{3}\right)$, where the reference angular velocity $\boldsymbol{\Omega}_{r}$ is defined by

$$
\boldsymbol{\Omega}_{r} \triangleq k_{g} \mathbf{e}_{3} \times \mathbf{R}^{\top} \mathbf{e}_{3}+\omega_{3 r} \mathbf{e}_{3}
$$

with $k_{g}>0$ positive gain and $\omega_{3 r} \in \mathbb{R}$ the reference yaw angular velocity to be specified by the outer-loop control. Note that $\dot{\omega}_{3 r}$ must be computable so that $\dot{\boldsymbol{\Omega}}_{r}$ is also computable by the torque controller.

- The outer-loop control defines the force control vector $\mathbf{F}_{c}$ together with the reference yaw angular velocity $\omega_{3 r}$ (for the inner-loop control) so as to fulfill the main objective of stabilizing $\mathbf{H}$ about $\mathbf{I}_{3}$ (or, equivalently, stabilizing $\left(\mathbf{e}_{p}, \mathbf{e}_{\Theta}\right)$ about zero), provided that the inner-loop control ensures the almost global asymptotic stability (almostGAS) and local exponential stability (LES) of the equi$\operatorname{librium}\left(\boldsymbol{\Omega}, \mathbf{R}^{\top} \mathbf{e}_{3}\right)=\left(\boldsymbol{\Omega}_{r}, \mathbf{e}_{3}\right)$.

The inner-loop control design is less involved and is postponed after the outer-loop control design.

\section{A. Outer-loop control design}

1) Force control design: Similarly to our prior work [9] we first proceed the control design for the stabilization of the visual error $\mathbf{e}_{p}$ defined by (8) about zero, but here without linear velocity measurements.

From (7), (8), it can be verified that the dynamics of $\mathbf{e}_{p}$ are

$$
\dot{\mathbf{e}}_{p}=-\boldsymbol{\Omega} \times\left(\mathbf{e}_{p}-\mathbf{m}^{\star}\right)+a^{\star} \mathbf{V}_{C}
$$


with $a^{\star} \triangleq \frac{\left(\mathbf{n}^{\star \top} \mathbf{m}^{\star}\right)}{d^{\star}}$ positive but unknown number. In the present work we consider the case of a downward-looking camera so that the condition $\mathbf{n}^{\star \top} \mathbf{m}^{\star}>0$ is satisfied when choosing $\mathbf{m}^{\star}=-\mathbf{e}_{3}$. As a result of the inner-loop control (to be designed thereafter) that ensures the almost-GAS and LES of $\left(\boldsymbol{\Omega}, \mathbf{R}^{\top} \mathbf{e}_{3}\right)=\left(\boldsymbol{\Omega}_{r}, \mathbf{e}_{3}\right), \boldsymbol{\Omega}$ converges to $\omega_{3 r} \mathbf{e}_{3}$ and, subsequently, $\Omega \times \mathbf{m}^{\star}$ converges to zero and $\mathbf{V}_{C}$ converges to $\mathbf{V}$. Therefore, (11) can be rewritten as

$$
\dot{\mathbf{e}}_{p}=-\boldsymbol{\Omega} \times \mathbf{e}_{p}+a^{\star} \mathbf{V}+\varepsilon(t)
$$

with the "disturbance" term $\varepsilon(t) \triangleq \boldsymbol{\Omega} \times \mathbf{m}^{\star}+a^{\star}\left(\mathbf{V}_{C}-\mathbf{V}\right)$ converging exponentially to zero.

From here, the outer-loop control design will be first carried out for the case of absence of sea currents (i.e. $\mathbf{v}_{f} \equiv \mathbf{0}$ ) so that the disturbance term $\boldsymbol{\Delta}_{F}$ involved in (5c) can be considered null, i.e. $\boldsymbol{\Delta}_{F} \equiv \mathbf{0}$. Then, we will show later on how to cope with sea currents by adding an integral correction action.

Proposition 1 Consider the dynamics of $\mathbf{e}_{p}$ given by (12), with the disturbance term $\varepsilon(t)$ remaining bounded for all time and converging exponentially to zero. Consider the translational dynamics $(5 \mathrm{c})$ with $\boldsymbol{\Delta}_{F} \equiv \mathbf{0}$. Introduce the augmented system

$$
\dot{\hat{\mathbf{e}}}_{p}=-\boldsymbol{\Omega} \times \hat{\mathbf{e}}_{p}-\mathbf{K}_{1} \hat{\mathbf{e}}_{p}+\mathbf{K}_{1} \mathbf{e}_{p}, \quad \hat{\mathbf{e}}_{p}(0) \in \mathbb{R}^{3}
$$

with $\mathbf{K}_{1} \in \mathbb{R}^{3 \times 3}$ positive matrix. Apply the control force

$$
\mathbf{F}_{c}=\bar{m} \mathbf{M}^{-1}\left(\operatorname{sat}^{\eta_{1}}\left(k_{2} \tilde{\mathbf{e}}_{p}\right)-\operatorname{sat}^{\eta_{2}}\left(k_{3} \mathbf{e}_{p}\right)\right)-\mathbf{F}_{g b}
$$

with positive numbers $\bar{m}, k_{2}, k_{3}, \eta_{1}, \eta_{2}$, and $\tilde{\mathbf{e}}_{p} \triangleq \hat{\mathbf{e}}_{p}$ $\mathbf{e}_{p}$, and $\operatorname{sat}^{(\cdot)}(\cdot)$ the classical saturation function. Assume that $\Omega$ remains bounded for all time. Then, the equilibrium $\left(\mathbf{e}_{p}, \hat{\mathbf{e}}_{p}, \mathbf{V}\right)=(\mathbf{0}, \mathbf{0}, \mathbf{0})$ is globally asymptotically stable (GAS). Moreover, $\mathbf{F}_{c}$ remains bounded by

$$
\left|\mathbf{F}_{c}(t)\right| \leq \bar{m} \underline{\lambda}_{\mathbf{M}}^{-1}\left(\eta_{1}+\eta_{2}\right)+\left|m g-F_{b}\right|
$$

with $\underline{\lambda}_{\mathbf{M}}$ the smallest eigenvalue of $\mathbf{M}$.

Proof: Since the perturbation term $\varepsilon(t)$ involved in (12) converges exponentially to zero as a result of the inner-loop control, according to the singular perturbation theory [11] one only needs to prove the asymptotic stability of the unperturbed system (i.e. setting $\varepsilon(t) \equiv 0$ in (12)). Consider the following Lyapunov function candidate

$$
\mathcal{L}=\int_{0}^{\left|\tilde{\mathbf{e}}_{p}\right|} \operatorname{sat}^{\eta_{1}}\left(k_{2} s\right) d s+\int_{0}^{\left|\mathbf{e}_{p}\right|} \text { sat }^{\eta_{2}}\left(k_{3} s\right) d s+\frac{a^{\star}}{2 \bar{m}}|\mathbf{M V}|^{2}
$$

One verifies that the time-derivative of $\mathcal{L}$ along any solution to the controlled unperturbed system is

$$
\begin{aligned}
\dot{\mathcal{L}}= & -\operatorname{sat}^{\eta_{1}}\left(k_{2} \tilde{\mathbf{e}}_{p}\right)^{\top} \mathbf{K}_{1} \tilde{\mathbf{e}}_{p} \\
& -a^{\star} \operatorname{sat}^{\eta_{1}}\left(k_{2} \tilde{\mathbf{e}}_{p}\right)^{\top} \mathbf{V}+a^{\star} \operatorname{sat}^{\eta_{2}}\left(k_{3} \mathbf{e}_{p}\right)^{\top} \mathbf{V} \\
& +a^{\star} \bar{m}^{-1}(\mathbf{M V})^{\top}\left(\mathbf{F}_{c}+\mathbf{F}_{g b}+\mathbf{F}_{d}(\mathbf{V})\right) \\
= & -\operatorname{sat}^{\eta_{1}}\left(k_{2} \tilde{\mathbf{e}}_{p}\right)^{\top} \mathbf{K}_{1} \tilde{\mathbf{e}}_{p}+\frac{a^{\star}}{\bar{m}}(\mathbf{M V})^{\top} \mathbf{F}_{d}(\mathbf{V}) \leq 0
\end{aligned}
$$

where the last inequality is obtained using the fact that $\mathbf{F}_{d}$ is a dissipative force so that $(\mathbf{M V})^{\top} \mathbf{F}_{d}(\mathbf{V}) \leq 0$. From (17) one deduces that $\hat{\mathbf{e}}_{p}$ converges to $\mathbf{e}_{p}$ and that $\mathbf{V}$ converges to zero. The convergence of $\dot{\mathbf{V}}$ to zero then follows. In view of (5c) with $\boldsymbol{\Delta}_{f} \equiv \mathbf{0}$ and $\mathbf{F}_{c}$ given by (14), one ensures that $\mathbf{e}_{p}$ converges to zero. The stability of the equilibrium $\left(\mathbf{e}_{p}, \hat{\mathbf{e}}_{p}, \mathbf{V}\right)=(\mathbf{0}, \mathbf{0}, \mathbf{0})$ is a direct consequence of (16) and (17). Finally, the bound of the force control vector $\mathbf{F}_{c}$ given by (15) is straightforwardly deduced from (14).

Remark 1 In first order approximations, the force control $\mathbf{F}_{c}$ given by (14) can be approximated by

$$
\mathbf{F}_{c}=k_{2} \bar{m} \mathbf{M}^{-1} \hat{\mathbf{e}}_{p}-\left(k_{2}+k_{3}\right) \bar{m} \mathbf{M}^{-1} \mathbf{e}_{p}-\mathbf{F}_{g b}
$$

The proof of GAS of the equilibrium $\left(\mathbf{e}_{p}, \hat{\mathbf{e}}_{p}, \mathbf{V}\right)=(\mathbf{0}, \mathbf{0}, \mathbf{0})$, when $\mathbf{F}_{c}$ is given by (18), proceeds identically to the proof of Proposition 1. The linear approximation (18) of $\mathbf{F}_{c}$ is useful for gain tuning using, for instance, pole placement technique, while the nonlinear expression (14) of $\mathbf{F}_{c}$ that involves saturation functions allows us to define explicitly the bound of the force control input $\mathbf{F}_{c}$ as given by (15). The latter property is of particular importance in practice since it is often desirable to take explicitly actuation limitations into account. For instance, (15) implies that the desired bound of $\left|\mathbf{F}_{c}\right|$ can be set to any value $\mu\left(>\left|m g-F_{b}\right|\right)$ if

$$
\eta_{1}+\eta_{2} \leq \bar{m}^{-1} \underline{\lambda}_{\mathbf{M}}\left(\mu-\left|m g-F_{b}\right|\right)
$$

It is noticeable that Proposition 1 only applies to the case where sea currents are negligible so that the perturbation term $\boldsymbol{\Delta}_{F}$ involved in (5c) can be considered as a null vector. As for the case of significant currents, it is necessary to enhance the control robustness by incorporating an integral correction action. However, in our case the system considered in Proposition 1 (i.e. $(13)+(12)+(5 c)$ ) is already a third-order time-varying system. Thus, adding an integrator would lead to a fourth-order time-varying system. Too high order system, together with the presence of an unknown multiplicative factor $a^{\star}$ in (12) and the unavailability of linear velocity measurements, excludes the possibility of establishing global (or semi-global) stability results similar to Proposition 1. However, it is still possible to state local exponential stability. For simplicity, let us consider the case where $\mathbf{M}$ can be roughly approximated by a positive diagonal matrix, i.e. $\mathbf{M} \approx \operatorname{diag}\left(m_{1,1}, m_{2,2}, m_{3,3}\right)$.

Proposition 2 Consider the dynamics of $\mathbf{e}_{p}$ given by (12) and consider the translational dynamics $(5 \mathrm{c})$ with constant disturbance $\boldsymbol{\Delta}_{F}$ and diagonal $\mathbf{M}=\operatorname{diag}\left(m_{1,1}, m_{2,2}, m_{3,3}\right)$. Introduce the following integrator

$$
\dot{\mathbf{z}}=-\boldsymbol{\Omega} \times \mathbf{z}+\mathbf{e}_{p}, \quad \mathbf{z}(0) \in \mathbb{R}^{3}
$$

and the following augmented system

$$
\dot{\hat{\mathbf{e}}}_{p}=-\boldsymbol{\Omega} \times \hat{\mathbf{e}}_{p}-\mathbf{K}_{1} \hat{\mathbf{e}}_{p}+\mathbf{K}_{1} \overline{\mathbf{e}}_{p}, \quad \hat{\mathbf{e}}_{p}(0) \in \mathbb{R}^{3}
$$

with $\overline{\mathbf{e}}_{p} \triangleq \mathbf{e}_{p}+k_{I} \mathbf{z}, k_{I}$ a positive integral gain, and $\mathbf{K}_{1} \in$ $\mathbb{R}^{3 \times 3}$ positive diagonal matrix. Apply the control force

$$
\mathbf{F}_{c}=\bar{m} \mathbf{M}^{-1}\left(\operatorname{sat}^{\eta_{1}}\left(k_{2} \tilde{\overline{\mathbf{e}}}_{p}\right)-\operatorname{sat}^{\eta_{2}}\left(k_{3} \overline{\mathbf{e}}_{p}\right)\right)-\mathbf{F}_{g b}
$$

with positive numbers $\bar{m}, k_{2}, k_{3}, \eta_{1}, \eta_{2}$, and $\tilde{\mathbf{e}}_{p} \triangleq \hat{\mathbf{e}}_{p}-\overline{\mathbf{e}}_{p}$. Choose $\eta_{2}$ high enough such that

$$
\eta_{2}>\bar{m}^{-1}\left|\mathbf{M} \boldsymbol{\Delta}_{F}\right|
$$

and choose $k_{I}$ satisfying

$$
k_{I}<\frac{k_{2} \underline{\lambda}_{\mathbf{K}_{1}}}{k_{2}+k_{3}}
$$


with $\underline{\lambda}_{\mathbf{K}_{1}}$ the smallest diagonal component of $\mathbf{K}_{1}$. Assume that the outer-loop control $\omega_{3 r}$ together with the innerloop control $\boldsymbol{\Gamma}_{c}$ ensures that $\boldsymbol{\Omega}$ can be considered as a first order term in first order approximations. Then, the equilibrium $\left(\hat{\mathbf{e}}_{p}, \mathbf{e}_{p}, \mathbf{V}, \mathbf{z}\right)=\left(k_{I} \mathbf{z}^{\star}, \mathbf{0}, \mathbf{0}, \mathbf{z}^{\star}\right)$, with $\mathbf{z}^{\star} \triangleq$ $\left(\bar{m} k_{3} k_{I}\right)^{-1} \mathbf{M} \boldsymbol{\Delta}_{F}$, of the controlled system is locally exponentially stable (LES).

Proof: One verifies that the linearized system of Eqs. (12), (5c), (20) augmented with integrator (19) around the equilibrium $\left(\hat{\mathbf{e}}_{p}, \mathbf{e}_{p}, \mathbf{V}, \mathbf{z}\right)=\left(k_{I} \mathbf{z}^{\star}, \mathbf{0}, \mathbf{0}, \mathbf{z}^{\star}\right)$ is $\dot{\mathbf{X}}=\mathbf{A X}$ with $\mathbf{X} \in \mathbb{R}^{12}$ and $\mathbf{A} \in \mathbb{R}^{12 \times 12}$ given by

$$
\begin{aligned}
& \mathbf{A} \triangleq\left[\begin{array}{cccc}
-\mathbf{K}_{1} & \mathbf{K}_{1} & \mathbf{0} & k_{I} \mathbf{K}_{1} \\
\mathbf{0} & \mathbf{0} & a^{\star} \mathbf{M}^{-1} & \mathbf{0} \\
k_{2} \bar{m} \mathbf{M}^{-1} & -\left(k_{2}+k_{3}\right) \bar{m} \mathbf{M}^{-1} & \mathbf{0} & -k_{I}\left(k_{2}+k_{3}\right) \bar{m} \mathbf{M}^{-1} \\
\mathbf{0} & \mathbf{I}_{3} & \mathbf{0} & \mathbf{0}
\end{array}\right]
\end{aligned}
$$

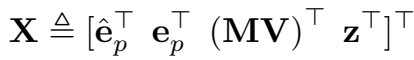

One verifies from $(5 \mathrm{c})$ and $(21)$ that at equilibrium configuration $\mathbf{z}^{\star} \triangleq\left(\bar{m} k_{3} k_{I}\right)^{-1} \mathbf{M} \boldsymbol{\Delta}_{F}$. Also from these equations, when $\tilde{\overline{\mathbf{e}}}_{p}=0$, if $\left|k_{2} \overline{\mathbf{e}}_{p}\right| \geq \eta_{2}$, one deduces that $\eta_{2}=\bar{m}^{-1}\left|\mathbf{M} \boldsymbol{\Delta}_{F}\right|$. Therefore condition (22) is required so that the integral action can compensate for the disturbance $\boldsymbol{\Delta}_{F}$.

The 12th-order characteristic polynomial of the linearized system is $Q(\lambda)=Q_{1}(\lambda) Q_{2}(\lambda) Q_{3}(\lambda)$, with

$$
\begin{aligned}
& Q_{i}(\lambda)=\lambda^{4}+k_{1 i} \lambda^{3}+\left(k_{2}+k_{3}\right) a^{\star} \frac{\bar{m}}{m_{i, i}^{2}} \lambda^{2} \\
& \quad+\left[k_{1 i} k_{3}+\left(k_{2}+k_{3}\right) k_{I}\right] a^{\star} \frac{\bar{m}}{m_{i, i}^{2}} \lambda+k_{1 i} k_{3} k_{I} a^{\star} \frac{\bar{m}}{m_{i, i}^{2}}
\end{aligned}
$$

with $k_{I}$ satisfying (23), direct application of Routh-Hurwitz criterion ensures the stability of the linearized system.

2) Control design of the reference yaw angular velocity $\omega_{3 r}$ : The previous part of outer-loop control design ensures the convergence of $\mathbf{e}_{p}$ to zero. From here, the design of $\omega_{3 r}$ can proceed identically to our prior work [9] and is, thus, recalled here for the sake of completeness.

Proposition 3 (see [9] for proof) Assume that the inner-loop torque control $\boldsymbol{\Gamma}_{c}$ ensures the almost-GAS and LES of the equilibrium $\left(\boldsymbol{\Omega}, \mathbf{R}^{\top} \mathbf{e}_{3}\right)=\left(\boldsymbol{\Omega}_{r}, \mathbf{e}_{3}\right)$, with $\boldsymbol{\Omega}_{r}$ defined by (10) combined with $\omega_{3 r}$ (involved in (10)) solution to the following system

$$
\dot{\omega}_{3 r}=-k_{\Theta 2} \omega_{3 r}-k_{\Theta 1} \mathrm{sat}^{\Delta_{\Theta}}\left(h_{1,2}\right), \quad \omega_{3 r}(0) \in \mathbb{R}
$$

with positive numbers $k_{\Theta 1}, k_{\Theta 2}, \Delta_{\Theta}$ and $h_{1,2}$ the element at the first row and second column of $\mathbf{H}$. Apply that the outerloop force control $\mathbf{F}_{c}$ given either by Proposition 1 (when $\boldsymbol{\Delta}_{F} \equiv \mathbf{0}$ ) or Proposition 2 (when $\boldsymbol{\Delta}_{F}$ is constant and non null). Then, the equilibrium $\mathbf{H}=\mathbf{I}_{3}$ is LES. Moreover, this equilibrium is almost-GAS in the case where $\mathbf{F}_{c}$ is given by Proposition 1 and $\boldsymbol{\Delta}_{F} \equiv \mathbf{0}$.

\section{B. Inner-loop control design}

The more involved part concerning the outer-loop control design has been presented. It remains to design an effective inner-loop torque control that ensures the stability of the equilibrium $\left(\boldsymbol{\Omega}, \mathbf{R}^{\top} \mathbf{e}_{3}\right)=\left(\boldsymbol{\Omega}_{r}, \mathbf{e}_{3}\right)$, with $\boldsymbol{\Omega}_{r}$ defined by (10) combined with (24).

In view of the rotation dynamics (i.e. (5b) and (5d)), it is not too difficult to carry out the above-mentioned objective since the sub-system under consideration is fully-actuated and the measurements of both $\boldsymbol{\Omega}$ and $\mathbf{R}^{\top} \mathbf{e}_{3}$ are at our disposal. However, the most troublesome term $\boldsymbol{\Delta}_{\Gamma}$ involved in $(5 \mathrm{~d})$ should be carefully addressed, especially when the vehicle is subjected to strong sea currents that excite the "Munk moment" effects. Since the angular velocity can be measured at high frequency and with good precision, we propose to estimate $\Delta_{\Gamma}$ using a high-gain observer similarly to the idea proposed in [10], [8, Proposition 8].

Lemma 2 Consider the following observer of $\boldsymbol{\Delta}_{\Gamma}$, assuming that $\boldsymbol{\Gamma}_{c}, \boldsymbol{\Omega}$, and $\mathbf{R}^{\top} \mathbf{e}_{3}$ are measured

$\left\{\begin{array}{l}\mathbf{J} \dot{\hat{\mathbf{\Omega}}}=(\mathbf{J} \boldsymbol{\Omega}) \times \hat{\boldsymbol{\Omega}}+\boldsymbol{\Gamma}_{c}+\boldsymbol{\Gamma}_{g}+\hat{\boldsymbol{\Delta}}_{\Gamma}+k_{0} \mathbf{J}(\boldsymbol{\Omega}-\hat{\boldsymbol{\Omega}}) \\ \dot{\hat{\boldsymbol{\Delta}}}_{\Gamma}=a_{0}^{2} k_{0}^{2} \mathbf{J}(\boldsymbol{\Omega}-\hat{\boldsymbol{\Omega}})\end{array}\right.$

with $\hat{\boldsymbol{\Omega}}$ and $\hat{\boldsymbol{\Delta}}_{\Gamma}$ the estimates of $\boldsymbol{\Omega}$ and $\boldsymbol{\Delta}_{\Gamma}$, respectively, $a_{0}, k_{0}$ some positive gains. Assume that $\dot{\Delta}_{\Gamma}$ is uniformly ultimately bounded (u.u.b.). Then for any $a_{0} \in(1-\sqrt{2} / 2,1+$ $\sqrt{2} / 2)$,

1) The errors $\hat{\boldsymbol{\Omega}}-\boldsymbol{\Omega}$ and $\hat{\boldsymbol{\Delta}}_{\Gamma}-\boldsymbol{\Delta}_{\Gamma}$ are u.u.b. by a positive constant $\varepsilon\left(k_{0}\right)$ that tends to zero when $k_{0}$ tends to $+\infty$. Moreover, these terms converge exponentially to zero for any $k_{0}>0$ if $\boldsymbol{\Delta}_{\Gamma}$ is constant.

2) $\hat{\boldsymbol{\Delta}}_{\Gamma}$ is u.u.b. by a constant independent of $k_{0}$.

The proof proceeds identically to the proof of [8, Proposition 8]. Now, we can use the estimate $\hat{\boldsymbol{\Delta}}_{\Gamma}$ as a feedforward term for the inner-loop torque control design.

Define the angular velocity error variable $\tilde{\Omega} \triangleq \boldsymbol{\Omega}-\boldsymbol{\Omega}_{r}$. From (5d), one obtains the following error equation

$$
\mathbf{J} \tilde{\tilde{\Omega}}=(\mathbf{J} \boldsymbol{\Omega})_{\times} \tilde{\boldsymbol{\Omega}}+\boldsymbol{\Gamma}_{c}+\boldsymbol{\Gamma}_{g}+\boldsymbol{\Gamma}+\hat{\boldsymbol{\Delta}}_{\Gamma}+\overline{\boldsymbol{\Delta}}_{\Gamma}
$$

with $\boldsymbol{\Gamma} \triangleq(\mathbf{J} \boldsymbol{\Omega})_{\times} \boldsymbol{\Omega}_{r}-\mathbf{J} \dot{\boldsymbol{\Omega}}_{r}$ and $\overline{\boldsymbol{\Delta}}_{\Gamma} \triangleq \boldsymbol{\Delta}_{\Gamma}-\hat{\boldsymbol{\Delta}}_{\Gamma}$.

Proposition 4 Consider error equation given by (26). Introduce the following integrator

Apply the control torque

$$
\dot{\mathbf{z}}_{\Omega}=\tilde{\Omega}, \mathbf{z}_{\Omega}(0) \in \mathbb{R}^{3}
$$

$$
\boldsymbol{\Gamma}_{c}=-\operatorname{sat}^{\eta_{3}}\left(\mathbf{K}_{\Omega} \tilde{\boldsymbol{\Omega}}\right)-\mathbf{K}_{i \Omega} \mathbf{z}_{\Omega}-\boldsymbol{\Gamma}-\hat{\boldsymbol{\Delta}}_{\Gamma}
$$

with $\mathbf{K}_{i \Omega}, \mathbf{K}_{\Omega} \in \mathbb{R}^{3 \times 3}$ positive diagonal gain matrices, $\eta_{3}$ a positive number, $\boldsymbol{\Omega}_{r}$ defined by (10) combined with (24), and with $\hat{\boldsymbol{\Delta}}_{\Gamma}$ given by Lemma 2 . Assume that $\boldsymbol{\Delta}_{\Gamma}$ is constant. Then, the following properties hold.

1) The error state $\left(\tilde{\mathbf{\Omega}}, \mathbf{z}_{\Omega}, \mathbf{R e}_{3}\right)$ converges either to $\left(\mathbf{0}, \mathbf{0}, \mathbf{e}_{3}\right)$ or $\left(\mathbf{0}, \mathbf{0},-\mathbf{e}_{3}\right)$ for all initial conditions.

2) The equilibrium $\left(\tilde{\mathbf{\Omega}}, \mathbf{z}_{\Omega}, \mathbf{R e}_{3}\right)=\left(\mathbf{0}, \mathbf{0}, \mathbf{e}_{3}\right)$ is almost-GAS and LES. The equilibrium $\left(\tilde{\mathbf{\Omega}}, \mathbf{z}_{\Omega}, \mathbf{R e}_{3}\right)=\left(\mathbf{0}, \mathbf{0},-\mathbf{e}_{3}\right)$ is unstable.

Proof: As a consequence of Lemma $2, \hat{\boldsymbol{\Delta}}_{\Gamma}$ converges exponentially to $\boldsymbol{\Delta}_{\Gamma}$ or, equivalently, $\bar{\Delta}_{\Gamma}$ converges exponentially to zero. Therefore, in view of the singular perturbation theory [11] it suffices to prove the asymptotic stability of the unperturbed system (i.e. setting $\bar{\Delta}_{\Gamma} \equiv \mathbf{0}$ in (26)). Consider the Lyapunov function candidate

$$
\mathcal{L}=\frac{1}{2} \tilde{\mathbf{\Omega}}^{\top} \mathbf{J} \tilde{\boldsymbol{\Omega}}+\operatorname{mgl}\left(1-\mathbf{e}_{3}^{\top} \mathbf{R}^{\top} \mathbf{e}_{3}\right)+\frac{1}{2} \mathbf{z}_{\Omega}^{\top} \mathbf{K}_{i \Omega} \mathbf{z}_{\Omega}
$$

Using error equation (26), the torque control expression (28), the definition (10) of $\Omega_{r}$, one verifies that the derivative of $\mathcal{L}$ satisfies 


$$
\begin{aligned}
\dot{\mathcal{L}}= & \tilde{\boldsymbol{\Omega}}^{\top}\left(-\operatorname{sat}^{\eta_{3}}\left(\mathbf{K}_{\Omega} \tilde{\boldsymbol{\Omega}}\right)-\mathbf{K}_{i \Omega} \mathbf{z}_{\Omega}\right) \\
& -m g l \boldsymbol{\Omega}_{r}^{\top} \mathbf{e}_{3} \times \mathbf{R}^{\top} \mathbf{e}_{3}+\mathbf{z}_{\Omega}^{\top} \mathbf{K}_{i \Omega} \tilde{\boldsymbol{\Omega}} \\
= & -\tilde{\boldsymbol{\Omega}}^{\top} \operatorname{sat}^{\eta_{3}}\left(\mathbf{K}_{\Omega} \tilde{\boldsymbol{\Omega}}\right)-m g l k_{g}\left|\mathbf{e}_{3} \times \mathbf{R}^{\top} \mathbf{e}_{3}\right|^{2} \leq 0
\end{aligned}
$$

Since $\dot{\mathcal{L}}$ is negative semi-definite, $\tilde{\boldsymbol{\Omega}}$ and $\mathbf{z}_{\Omega}$ are bounded w.r.t. initial conditions. Since $\boldsymbol{\Omega}_{r}$ and its derivative are bounded thanks to the expressions (10) and (24), one deduces from (26) that $\dot{\tilde{\Omega}}$ is also bounded. Then it is straightforward to verify that $\ddot{\mathcal{L}}$ is also bounded, implying the uniform continuity of $\dot{\mathcal{L}}$. Then, application of Barbalat's lemma ensures that $\dot{\mathcal{L}}$ and, thus, $\tilde{\boldsymbol{\Omega}}$ and $\mathbf{e}_{3} \times \mathbf{R}^{\top} \mathbf{e}_{3}$ converge to zero. Next, using Barbalatlike arguments it can be shown that $\dot{\tilde{\Omega}}$ also converges to zero, implying the convergence of $\mathbf{z}_{\Omega}$ to zero. The convergence of $\mathbf{e}_{3} \times \mathbf{R}^{\top} \mathbf{e}_{3}$ to zero implies that $\mathbf{R e}_{3}$ converges to either $\mathbf{e}_{3}$ or $-\mathbf{e}_{3}$. So far we have proved that $\left(\tilde{\boldsymbol{\Omega}}, \mathbf{z}_{\Omega}, \mathbf{R e}_{3}\right)$ converges either to $\left(\mathbf{0}, \mathbf{0}, \mathbf{e}_{3}\right)$ or $\left(\mathbf{0}, \mathbf{0},-\mathbf{e}_{3}\right)$.

It remains to show that the "desired" equilibrium $\left(\tilde{\boldsymbol{\Omega}}, \mathbf{z}_{\Omega}, \mathbf{R e}_{3}\right)=\left(\mathbf{0}, \mathbf{0}, \mathbf{e}_{3}\right)$ is LES and the "undesired" equilibrium $\left(\tilde{\boldsymbol{\Omega}}, \mathbf{z}_{\Omega}, \mathbf{R e}_{3}\right)=\left(\mathbf{0}, \mathbf{0},-\mathbf{e}_{3}\right)$ is unstable. Note that the almost-GAS of the "desired" equilibrium then directly follows. In the first-order approximations, one has $\mathbf{R} \approx \mathbf{I}+\boldsymbol{\Theta}_{\times}$with $\boldsymbol{\Theta}=[\phi, \theta, \psi]^{\top}$ and, subsequently, $\mathbf{e}_{3 \times} \mathbf{R}^{\top} \mathbf{e}_{3} \approx[-\phi,-\theta, 0]^{\top}$. Denoting $\left[\tilde{\omega}_{1}, \tilde{\omega}_{2}, \tilde{\omega}_{3}\right]^{\top} \triangleq \tilde{\boldsymbol{\Omega}}$ and using the approximation $\dot{\Theta} \approx \Omega$, one obtains the following linearized system of (26) and (27)

$\left\{\begin{array}{l}\dot{\phi} \approx \tilde{\omega}_{1}-k_{g} \phi \\ \dot{\theta} \approx \tilde{\omega}_{2}-k_{g} \theta \\ \dot{\tilde{\mathbf{\Omega}}} \approx-\mathbf{J}^{-1} \mathbf{K}_{\Omega} \tilde{\mathbf{\Omega}}-\mathbf{J}^{-1} \mathbf{K}_{i \Omega} \mathbf{z}_{\Omega}+m g l \mathbf{J}^{-1}[-\phi,-\theta, 0]^{\top} \\ \dot{\mathbf{z}}_{\Omega}=\tilde{\mathbf{\Omega}}\end{array}\right.$

Consider the following Lyapunov function candidate

$$
\mathcal{L}_{\Omega}=\frac{1}{2} \tilde{\boldsymbol{\Omega}}^{\top} \mathbf{J} \tilde{\boldsymbol{\Omega}}+\frac{1}{2} \mathbf{z}_{\Omega}^{\top} \mathbf{K}_{i \Omega} \mathbf{z}_{\Omega}+\frac{1}{2} m g l\left(\phi^{2}+\theta^{2}\right)
$$

One verifies that

$$
\dot{\mathcal{L}}_{\Omega}=-\tilde{\boldsymbol{\Omega}}^{\top} \mathbf{K}_{\Omega} \tilde{\boldsymbol{\Omega}}-k_{g} m g l\left(\phi^{2}+\theta^{2}\right) \leq 0
$$

From here, LaSalle's principle ensures that $\tilde{\Omega}, \phi$ and $\theta$ and, thus, $\dot{\tilde{\Omega}}$ converge to zero, which implies the convergence of $\mathbf{z}_{\Omega}$ to zero. The convergence of $\phi$ and $\theta$ to zero is equivalent to the convergence of $\mathbf{R}^{\top} \mathbf{e}_{3}$ to $\mathbf{e}_{3}$. Since the equilibrium $\left(\tilde{\mathbf{\Omega}}, \mathbf{z}_{\Omega}, \mathbf{R}^{\top} \mathbf{e}_{3}\right)=\left(\mathbf{0}, \mathbf{0}, \mathbf{e}_{3}\right)$ of the linearized system (31) is asymptotically stable, it is also exponentially stable.

Now, the Chetaev's theorem is used to prove the instability of the equilibrium $\left(\tilde{\boldsymbol{\Omega}}, \mathbf{z}_{\Omega}, \mathbf{R}^{\top} \mathbf{e}_{3}\right)=\left(\mathbf{0}, \mathbf{0},-\mathbf{e}_{3}\right)$. Define $\mathbf{y}=$ $\mathbf{e}_{3}+\mathbf{R}^{\top} \mathbf{e}_{3}$. Consider the positive function $\mathcal{S}_{1}(\mathbf{y}) \triangleq \mathbf{y}^{\top} \mathbf{e}_{3}=$ $1+\mathbf{e}_{3}^{\top} \mathbf{R}^{\top} \mathbf{e}_{3}$, satisfying $\mathcal{S}_{1}(\mathbf{0})=0$. Define $U_{r} \triangleq\left\{\mathbf{y} \mid \mathcal{S}_{1}(\mathbf{y})>\right.$ $0,|\mathbf{y}|<r\}$ for some number $0<r<1$. Note that $U_{r}$ is nonempty. By neglecting all high-order terms, one verifies that

$$
\dot{\mathcal{S}}_{1} \approx \mathbf{e}_{3}^{\top} \mathbf{R} \boldsymbol{\Omega}_{r \times} \mathbf{e}_{3}=k_{g}\left|\mathbf{e}_{3 \times} \mathbf{R}^{\top} \mathbf{e}_{3}\right|^{2}=k_{g}\left|\mathbf{e}_{3 \times} \mathbf{y}\right|^{2}
$$

\begin{tabular}{|c|c|c|}
\hline Specification & \multicolumn{2}{|c|}{ Numerical value } \\
\hline$m[k g]$ & \\
\hline$F_{b}[N]$ & \multicolumn{2}{|l|}{$1.01 \mathrm{mg}$} \\
\hline$l[m]$ & \multicolumn{2}{|c|}{0.025} \\
\hline $\mathbf{r}_{C}[\mathrm{~m}]$ & \multicolumn{2}{|c|}{$\left[\begin{array}{lll}0 & 0 & 0.15\end{array}\right]$} \\
\hline & $0.0842 \quad 0.004$ & 0.005 \\
\hline $\mathbf{J}_{0}\left[\mathrm{~kg} \cdot \mathrm{m}^{2}\right]$ & 0.2643 & 0.007 \\
\hline & 0.007 & 0.3116 \\
\hline \multirow{3}{*}{$\mathbf{M}_{A}^{22}\left[k g \cdot m^{2}\right]$} & 0.005 & $0.006]$ \\
\hline & 0.005 & 0.008 \\
\hline & 0.008 & 0.3 \\
\hline \multirow{3}{*}{$\mathbf{M}_{A}^{11}[k g]$} & {$\left[\begin{array}{ll}1.39 & 0.10\end{array}\right.$} & 0.12 \\
\hline & 4.26 & 0.13 \\
\hline & 0.12 & 4.02 \\
\hline \multirow{3}{*}{$\mathbf{M}_{A}^{21}=\mathbf{M}_{A}^{12}$} & 0.002 & 0.01 \\
\hline & 0.003 & 0.018 \\
\hline & {$\left[\begin{array}{ll}0.01 & 0.018\end{array}\right.$} & 0.003 \\
\hline $\mathbf{D}_{V l}\left[k g \cdot s^{-1}\right]$ & \multicolumn{2}{|c|}{$\operatorname{diag}(5.85,9.21,11.03)$} \\
\hline $\mathbf{D}_{V q}\left[\mathrm{~kg} \cdot \mathrm{m}^{-1}\right]$ & \multicolumn{2}{|c|}{$\operatorname{diag}(36.57,57.58,68.97)$} \\
\hline $\mathbf{D}_{\Omega l}\left[\mathrm{~kg} \cdot \mathrm{m}^{2} \cdot \mathrm{s}^{-1}\right]$ & \multirow{2}{*}{\multicolumn{2}{|c|}{$\begin{array}{c}\operatorname{diag}(0.01126,0.01855,0.01701) \\
\operatorname{diag}(0.0053,0.0130,0.0118)\end{array}$}} \\
\hline $\mathbf{D}_{\Omega q}[N . m]$ & & \\
\hline$\hat{\mathbf{M}}=m \mathbf{I}_{3}+\hat{\mathbf{M}}_{A}^{11}[k g]$ & \multicolumn{2}{|c|}{$\operatorname{diag}(8.712,12.712,10.816)$} \\
\hline$\hat{\mathbf{J}}=\mathbf{J}_{0}+\hat{\mathbf{M}}_{A}^{22}\left[k g \cdot m^{2}\right]$ & \multicolumn{2}{|c|}{$\operatorname{diag}(0.1642,0.5643,0.5116)$} \\
\hline$\hat{\mathbf{D}}=m l \mathbf{e}_{3 \times}[\mathrm{kg} . \mathrm{m}]$ & \multicolumn{2}{|c|}{$0.19 \mathbf{e}_{3 \times}$} \\
\hline
\end{tabular}

For all $\mathbf{y} \in U_{r}$, the fact that $\mathbf{y}^{\top} \mathbf{e}_{3}>0$ is equivalent to $\left|\mathbf{e}_{3 \times} \mathbf{y}\right|^{2}>0$, which implies that $\dot{\mathcal{S}}_{1}>0$. Since all conditions of Chetaev's theorem are now united [11], the origin of the linearized system about the undesired equilibrium (so that $\mathbf{y}=\mathbf{0})$ is unstable.
TABLE I

SPECIFICATIONS OF THE AUV

\begin{tabular}{|c|c|}
\hline Controller & Gains and other parameters \\
\hline Proposition 2 & $\mathbf{K}_{1}=\operatorname{diag}(3 s, 3 s, 3 s)$ \\
& $k_{2}=\frac{8}{3} \frac{s^{2}}{a^{\star}}, k_{3}=\frac{1}{3} \frac{s^{2}}{a^{\star}}, s=\sqrt{2}$ \\
& $k_{I}=0.7, \eta_{1}=1.8, \eta_{2}=2.3$ \\
\hline Proposition 3 & $k_{g}=1, k_{\Theta 1}=\sqrt{2}, k_{\Theta 2}=1 / 2, \Delta_{\Theta}=1$ \\
\hline Proposition 4 & $\mathbf{K}_{\boldsymbol{\Omega}}=\operatorname{diag}(3,3,3), \mathbf{K}_{i \Omega}=0.7 \mathbf{K}_{\boldsymbol{\Omega}}$ \\
& $a_{0}=0.5, k_{0}=20, \eta_{3}=8$ \\
\hline
\end{tabular}

CONTROL GAINS AND PARAMETERS

\section{Simulation RESUlts}

The proposed control approach has been tested in simulation using a realistic model of a fully-actuated AUV with compact shape similarly to the BlueROV acquired by our team [1]. The AUV's physical parameters are given in Table I. The robustness of the proposed controller w.r.t. to model uncertainties are tested by using the "erroneous" estimated parameters $\hat{\mathbf{J}}, \hat{\mathbf{M}}, \hat{\mathbf{D}}$ given in Table I instead of the corresponding true values.

The homography $\mathbf{H}$ is directly computed using (6) with $d^{\star}=1(m)$ and $\mathbf{n}^{\star}=\mathbf{R}_{\left\{\frac{\pi}{18}, \frac{\pi}{6}, 0\right\}}\left(-\mathbf{e}_{3}\right)=$ $[-0.4924,0.1736,-0.8529]^{\top}$. The initial conditions are chosen as follows: $\mathbf{p}_{C}(0)=[-2,-1.5,-1]^{\top}(m), \mathbf{R}(0)=$ $\mathbf{R}_{\left\{\frac{\pi}{18},-\frac{\pi}{18}, \pi\right\}}, \mathbf{V}(0)=\boldsymbol{\Omega}(0)=\mathbf{0}$. The initial yaw error is very large, i.e. $\psi=\pi$, in order to verify the large stability domain of the proposed controller. For all presented simulations, the outer-loop controller defined in Propositions 2 and 3 and the inner-loop controller defined in Proposition 4 are simulated. Control parameters and gains, obtained from the classical pole placement technique, are summarized in Table II.

\section{A. Simulation $1-$ Performance in absence of current $\mathbf{v}_{f}=\mathbf{0}$}

The first simulation is dedicated to show the performance of the controller when sea current is absent. The integrators are disable by setting $k_{I}=0$ and $\mathbf{K}_{i \Omega}=\mathbf{0}$. Simulation results are reported in Figs. 3 - 6. The time evolutions of the vehicle's 
position and orientation are reported in Fig. 3. One observes that the position and orientation errors converge smoothly to zero despite large initial yaw error. Fig. 4 shows the fast convergence of the visual error estimate $\hat{\mathbf{e}}_{p}$ to $\mathbf{e}_{p}$. Then, both of them converge to zero together after about 10 seconds. The time evolutions of the control force and torque are shown in Fig. 5. All components of control force and torque converge to zero except the third component of the control force that allows to compensate for $\mathbf{F}_{g b}$. The convergence of angular velocity $\boldsymbol{\Omega}$ to the reference $\boldsymbol{\Omega}_{r}$ in Fig. 6 shows the effectiveness of the inner-loop control.
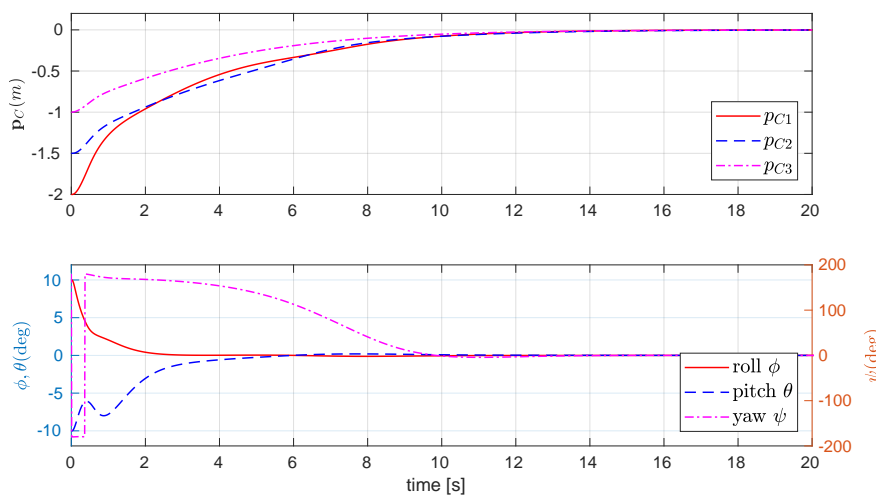

Fig. 3. AUV's position and attitude (Euler angles) vs. time (Simulation 1)
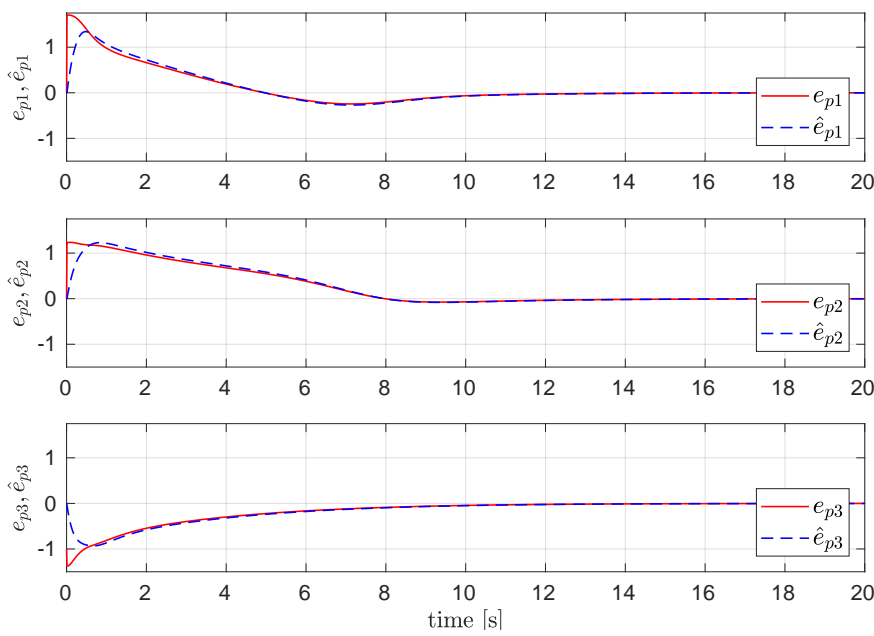

Fig. 4. $\mathbf{e}_{p}$ and $\hat{\mathbf{e}}_{p}$ vs. time (Simulation 1)
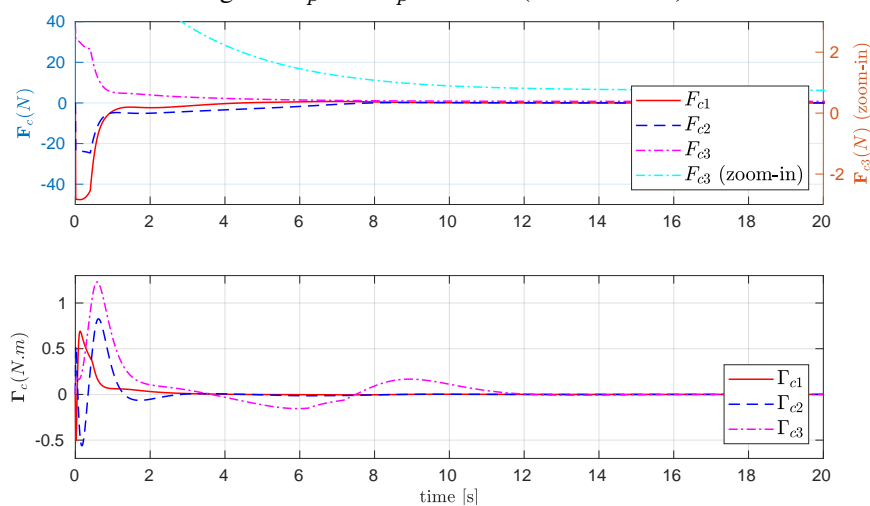

Fig. 5. Control force $\mathbf{F}_{c}$ (smaller scale on the right side for visibility of $F_{c 3}$ ) and moment $\boldsymbol{\Gamma}_{c}$ vs. time (Simulation 1)
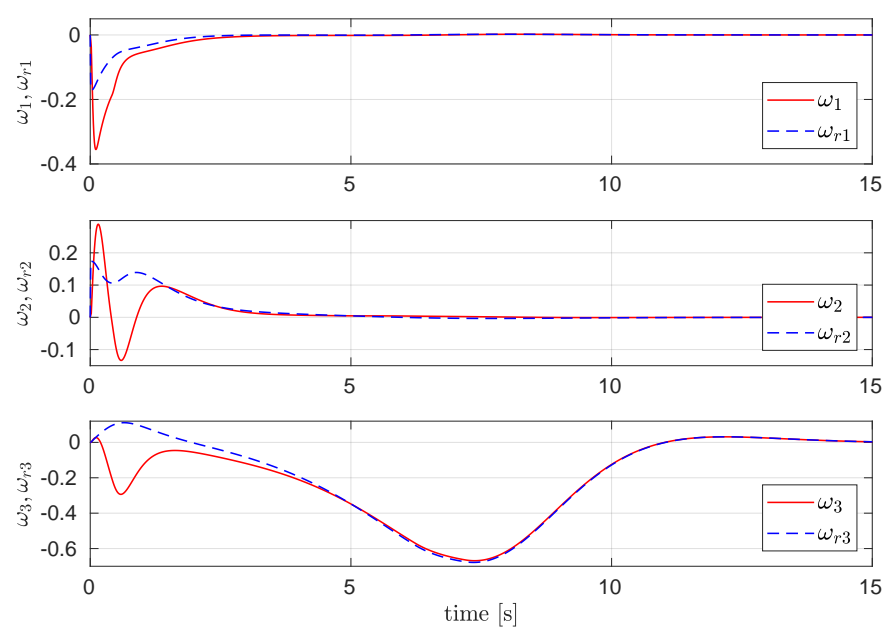

Fig. 6. $\boldsymbol{\Omega}$ and $\boldsymbol{\Omega}_{r}$ vs. time (Simulation 1)

B. Simulation 2 - Performance with current $\mathbf{v}_{f}=$ $\left[\frac{1}{2 \sqrt{2}}, \frac{1}{2 \sqrt{2}}, 0\right]^{\top}(m / s)$

In this simulation, a constant current velocity $\mathbf{v}_{f}=$ $\left[\frac{1}{2 \sqrt{2}}, \frac{1}{2 \sqrt{2}}, 0\right]^{\top}(\mathrm{m} / \mathrm{s})$ is introduced. The drag force $\mathbf{F}_{d}(\mathbf{V})$ and "Munk moment" are no longer negligible like in the previous simulation. However, thanks to the estimate $\hat{\Delta}_{\Gamma}$ and the integral actions in both inner-loop and outer-loop controls, the controller manages to compensate for the drag force and Munk moment as well as other model errors. The AUV's position and orientation converge to zero with quite good behaviour and small overshoot (see Fig. 7). The effect of the sea current can be seen on the estimate terms $\hat{\mathbf{e}}_{p}, \hat{\Delta}_{\Gamma}$ and the integral term $\mathbf{z}$. Since the current is on horizontal plane with including surge and sway components, one observes in Figs. 8-10 that $\hat{e}_{p 1}, \hat{e}_{p 2}, z_{1}, z_{2}$ and $\hat{\Delta}_{\Gamma 3}$ converge to non-null values. The control force and torque vectors converge to the values dictated by the compensation of the current and $\mathbf{F}_{g b}$ as shown in Fig. 9. The inner-loop control with asymptotic convergence of angular velocity $\Omega$ to the reference $\Omega_{r}$ is shown in Fig. 11. Also from this figure, one observes the convergence of $\omega_{r 1}$ and $\omega_{r 2}$ to zero, implying the convergence of $\mathbf{R}^{\top} \mathbf{e}_{3}$ to $\mathbf{e}_{3}$.

\section{CONCLUSIONS}

We have proposed an inertial-aided homography-based visual servo control approach for the stabilization of compact fully-actuated AUVs without relying on linear velocity measurements. The advanced features of our approach lie in the fact that both linear velocity measurements and homography decomposition are not required. A testing campaign with a real AUV is now envisioned to validate the proposed approach in challenging sea environment.

\section{REFERENCES}

[1] http://bluerobotics.com/store/retired/bluerov-r1/.

[2] S. Benhimane and E. Malis. Homography-based $2 d$ visual tracking and servoing. Int. J. of Robotics Research, 26(7):661-676, 2007.

[3] L. Brignone, M. Perrier, and C. Viala. A fully autonomous docking strategy for intervention AUVs. In MTS/IEEE OCEANS'07, pages 1-6, 2007. 

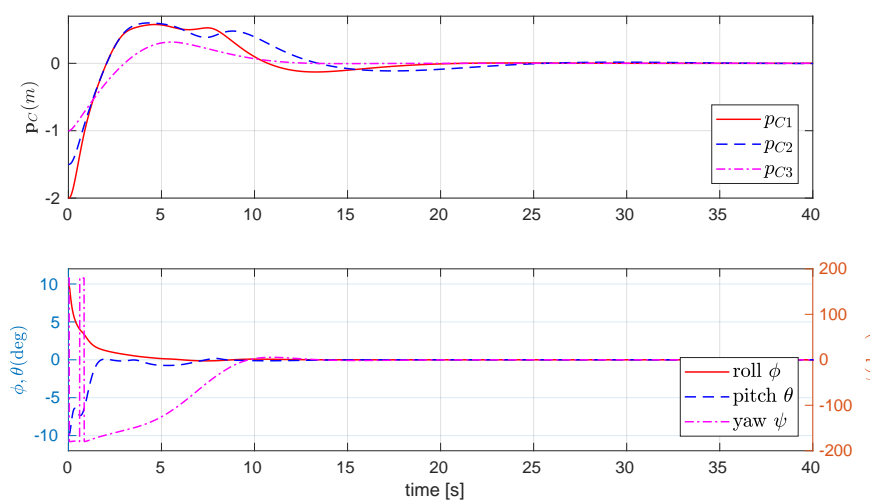

Fig. 7. AUV's position and attitude (Euler angles) vs. time (Simulation 2)
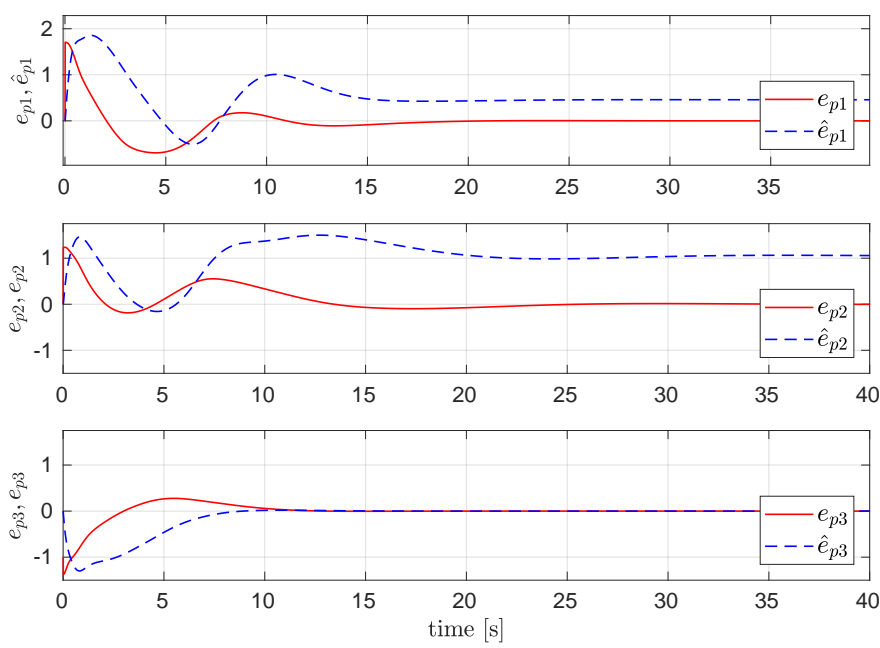

Fig. 8. $\mathbf{e}_{p}$ and $\hat{\mathbf{e}}_{p}$ vs. time (Simulation 2)
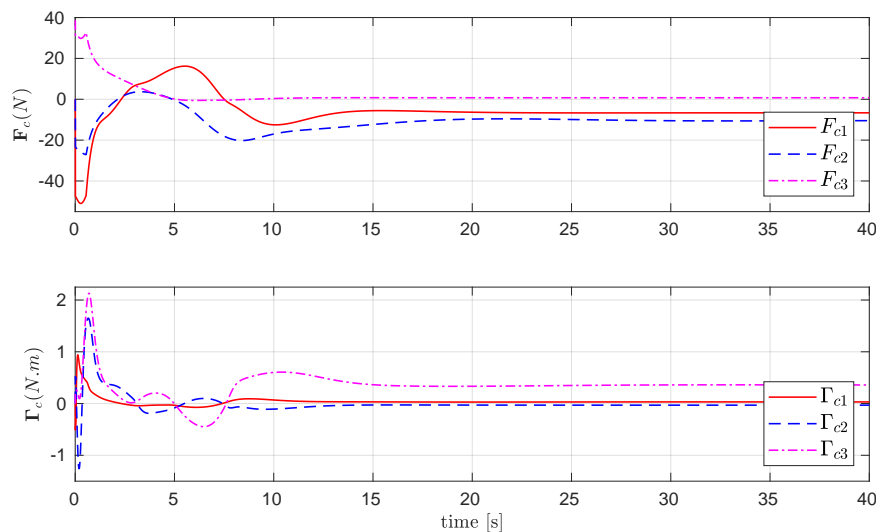

Fig. 9. Control force $\mathbf{F}_{c}$ and moment $\boldsymbol{\Gamma}_{c}$ vs. time (Simulation 2)

[4] F. Chaumette and S. Hutchinson. Visual servo control, Part II: Advanced approaches. IEEE Robotics and Automation Mag., 14(1):109-118, 2007.

[5] T. I. Fossen. Marine Control Systems. Marine Cybernetix AS, 2002.

[6] R. Garcia, J. Batlle, X. Cufi, and J. Amat. Positioning an underwater vehicle through image mosaicking. In IEEE Int. Conf. on Robotics \& Automation, 2001.

[7] T. Hamel and R. Mahony. Visual servoing of an under-actuated dynamic rigid-body system: an image-based approach. IEEE Trans. on Robotics and Automation, 18(2):187-198, 2002.

[8] M.-D. Hua. Contributions to the automatic control of aerial vehicles. PhD thesis, 2009.

[9] M.-D. Hua, G. Allibert, S. Krupínski, and T. Hamel. Homographybased visual servoing for autonomous underwater vehicles. In IFAC World Congress, pages 5729-5733, 2014.

[10] M.-D. Hua, T. Hamel, P. Morin, and C. Samson. Balanced-force-control
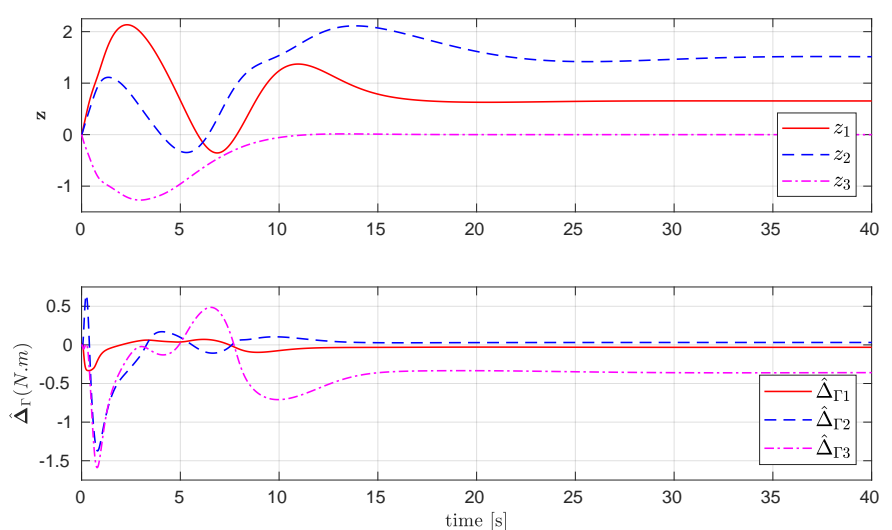

Fig. 10. $\mathbf{z}$ and $\hat{\boldsymbol{\Delta}}_{\Gamma}$ vs. time (Simulation 2)
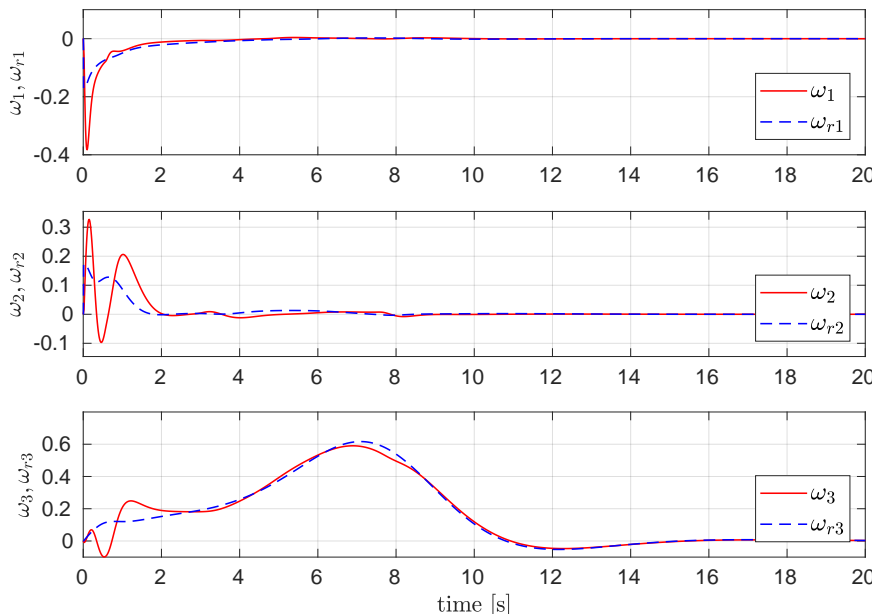

Fig. 11. $\boldsymbol{\Omega}$ and $\boldsymbol{\Omega}_{r}$ vs. time (Simulation 2)

of underactuated thrust-propelled vehicles. 46th IEEE Conference on Decision and Control (CDC), pages 6435-6441, 2007.

[11] H. K. Khalil. Nonlinear systems (3rd Edition). Prentice Hall, 2002.

[12] S. Krupínski, G. Allibert, M.-D. Hua, and T. Hamel. Pipeline tracking for fully-actuated autonomous underwater vehicle using visual servo control. In American Control Conference (ACC), pages 6196-6202, 2012.

[13] S. Krupínski, G. Allibert, M.-D. Hua, and T. Hamel. An inertialaided homography-based visual servoing control approach for (almost) fully actuated autonomous underwater vehicles. Accepted for IEEE Transactions on Robotics, 2017.

[14] N. E. Leonard. Stability of a bottom-heavy underwater vehicle. Automatica, 33(3):331-246, 1997.

[15] J.-E Lots, D. M. Lane, E. Trucco, and F. Chaumette. A 2-D visual servoing for underwater vehicle station keeping. In IEEE Int. Conf. on Robotics and Automation (ICRA), pages 2767-2772, 2001.

[16] E. Malis, F. Chaumette, and S. Boudet. $21 / 2 \mathrm{D}$ visual servoing. IEEE Trans. on Robotics and Automation, 15(2):238-250, 1999.

[17] E. Malis and M. Vargas. Deeper understanding of the homography decomposition for vision-based control. INRIA Researh report, RR6303:90, 2007

[18] N. Palomeras, S. Nagappa, D. Ribas, N. Gracias, and M. Carreras Vision-based localization and mapping system for AUV intervention. In MTS/IEEE OCEANS'13, pages 1-7, 2013

[19] H. Plinval, P. Morin, P. Mouyon, and T. Hamel. Visual servoing for underactuated VTOL UAVs: a linear, homography-based framework. Int. J. of Robust and Nonlinear Control, 24(16):2285-2308, 2014.

[20] P. Rives and J.-J. Borrelly. Underwater pipe inspection task using visual servoing techniques. In IEEE Int. Conf. on Intelligent Robots and Systems (IROS), pages 63-68, 1997.

[21] S. van der Zwaan, A. Bernardino, and J. Santos-Victor. Visual station keeping for floating robots in unstructured environments. Robotics and Autonomous Systems, 39(3):145-155, 2002. 